\title{
Peces mesopelágicos frente a la costa norte de Chile $\left(18^{\circ} 25^{\prime}-21^{\circ} 47^{\prime} S\right)^{1}$
}

\author{
Walter Sielfeld K., Mauricio Vargas F. y Rosalino Fuenzalida F. \\ Departamento de Ciencias del Mar \\ Universidad Arturo Prat \\ Casilla 121, Iquique, Chile
}

RESUMEN. Se estudian los peces mesopelágicos capturados en la capa profunda de dispersión sónica (DSL), en cinco transectos ubicados entre los $18^{\circ} 25^{\prime}$ y $21^{\circ} 47^{\prime} \mathrm{S}$ frente a las costas de la I Región de Chile. Los transectos se extendieron desde las 10 a las 100 millas de la costa y las capturas se realizaron mediante una red de ti po IKMT de $9,16 \mathrm{~m}^{2}$ de boca, en septiembre de 1988. Conjuntamente se registró la DSL mediante un equipo SIMRAD (32 KHz de frecuencia) y las condiciones de temperatura y salinidad de cada: estación.

En el área y período de estudio se detectaron 39 especies y 21 familias. En términos de biomasa la familia más importante fue Myctophidae (22\%), mientras que en número de individuos destacó Gonostomatidae (67\%). El 62\% de la ictiocenosis correspondió a especies de aguas temperadas (tropicales y/o subtropicales) y un 7,7\% a especies de aguas frías (antárticas y/o subantárticas), sin que se pierda el carácter transicional de la comunidad. El análisis de diversidad de Shannon- Wiener y de similitud de Winer mostró la existencia de 4 grupos discretos de estaciones, determinados por condiciones oceanográficas y masas de agua particulares. La DSL mostró un descenso hasta los $250 \mathrm{~m}$ de profundidad durante el día y la separación de una capa que asciende hasta la superficie durante la noche.

Palabras claves: ictiofauna mesopelágica, capa profunda de dispersión sónica, características oceanográficas, norte de Chile.

\section{Mesopelagic fishes off northern Chile $\left(1^{\circ} 25^{\prime}-21^{\circ} 47^{\prime} \mathrm{S}\right)$}

\begin{abstract}
Mesopelagic fishes captured in the deep scattering layer (DSL) off the I Region of Chile, in 5 transects between $18^{\circ} 25^{\prime}$ and $21^{\circ} 47^{\prime} \mathrm{S}$, from 10 to 100 miles offshore, were studied. A $9.16 \mathrm{~m}^{2}$ mouth IKMT net was used. The DSL was registered with a SIMRAD ecosounder at frecuencies of $32 \mathrm{Khz}$ and jointly the oceanographic conditions at each station were recorded.

In 23 sampling stations 20 families and 39 species of fishes were detected. Most abundant families were Myctophidae and Gonostomatidae, the former with $22 \%$ of total fish biomass and the latter $67 \%$ of total captured specimens. $62 \%$ of the identified species were temperate water elements (tropical andlor subtropical) and 7,7\% cold water species (antarctic andl or subantarctic), showing that the community structure is transitional between tropical and subantartic communities. The Shannon-Wiener index (diversity) and Winer index (similarity) showed the existence of four discrete groups of stations, determined by the oceanographic conditions and water masses. The DSL showed an upper limit at $250 \mathrm{~m}$ during the day and the separation of a layer that ascended to the surface during the night.
\end{abstract}

Key words: mesopelagic fishes, deep scattering layer, oceanographic characteristics, northern Chile.

\section{INTRODUCCION}

Los peces de media agua conforman por 10 general agregaciones numerosas, debido a 10 cual representan una fuente alternativa potencial, por ejemplo para la producción masiva de harina de pescado. Gjosaeter y Kawaguchi (1980) estiman un standing stock de 51 millones de toneladas para el Pacífico suroriental; además entregan un resumen de densidades de peces mesopelágicos en distintos océanos

\footnotetext{
1 Estudio financiado por el proyecto FAO-PNUD CHI/87/007.
} 
y regiones. Por otra parte, una recopilación y análisis de información nacional e internacional sobre distintos aspectos biológicos y pesqueros de estos peces es realizada por Acuña (1986).

Los peces mesopelágicos del Pacífico suroriental chileno han sido objeto de otros estudios, por parte de: Craddock y Mead (1970) sobre especies capturadas frente a Valparaíso; Parín et al. (1980) sobre capturas realizadas por la flota pesquera rusa entre los $19^{\circ}$ y $25^{\circ} \mathrm{S}$; Kong y Meléndez (1991) sobre especies capturadas entre Arica e Isla Mocha; y sobre la familia Myctophidae en particular (Wisner, 1976; Sáez, 1982; Acuña, 1986).

En el marco del proyecto FAO-PNUD CHI/87/ 007 «Aplicación de nuevas tecnologías para captura de recursos hidrobiológicos marinos no tradicionales en la I Región de Chile», se realizó el crucero «Prospección de recursos mesopelágicos», que incluyó el estudio de variables oceanográficas físicas con el fin de analizar su relación con estos recursos vivos y la estructura de las masas de agua.

El presente estudio tiene por objeto caracterizar la comunidad de peces mesopelágicos que habita frente a las costas del extremo norte de Chile ( $18^{\circ} 25^{\prime}$ $\left.21^{\circ} 47^{\prime} \mathrm{S}\right)$, considerando la distribución, abundancia relativa y diversidad, en asociación con las características oceanográficas observadas, durante septiembre de 1988.

\section{MATERIALES Y METODOS}

\section{Estaciones de muestreo}

Del 3 al 12 de septiembre de 1988 se efectuó, con el B/I «Carlos Porter» del Instituto de Fomento Pesquero (IFOP), un crucero de prospección entre los $18^{\circ} 25^{\prime}$ y $21^{\circ} 47^{\prime} \mathrm{S}$, hasta las 100 millas de la costa, incluyendo 23 estaciones en 5 transectos (Fig. 1; Tabla 1).

\section{Muestreo y análisis de condiciones oceanográficas}

El estudio oceanográfico consideró el registro de la temperatura y salinidad en cada una de las estaciones de muestreo, entre la superficie y los $1.000 \mathrm{~m}$ de profundidad. Las muestras se obtuvieron con botellas Nansen provistas de termómetros de inversión. Para la determinación de la salinidad se utilizóun salinómetro Autolab Modelo 601, y para el cálculo de la densidad se usó la ecuación de estado in- ternacional para el agua de mar, de Millero y Poisson (1981). La información fue procesada y graficada para analizar la distribución superficial de temperatura, salinidad y densidad. Además, las masas de agua presentes en los niveles de muestreo se analizaron utilizando el triángulo de mezcla utilizado por Kelly et al. (1988).

La denominación de las masas de agua del Pacífico suroriental consideró el criterio de Knox (1970), replanteado posteriormente por Arana y Gaete (1983), que frente a Sudamérica reconoce aguas ecuatoriales, tropicales, subtropicales, subantárticas y antárticas. Las masas de agua asociadas a la costa norte y central de Chile representan, sin embargo, zonas transicionales entre las masas de aguas ecuatoriales y subantárticas, gracias a sus propiedades físicas (Sverdrup et al., 1942) y biológicas (Ebeling, 1962)

\section{Muestreo y análisis de la ictiofauna mesopelágica}

La pesca de media agua se realizó con una red tipo IKMT (Isaac Kid Midwater Trawl), de 9,16 $\mathrm{m}^{2}$ de

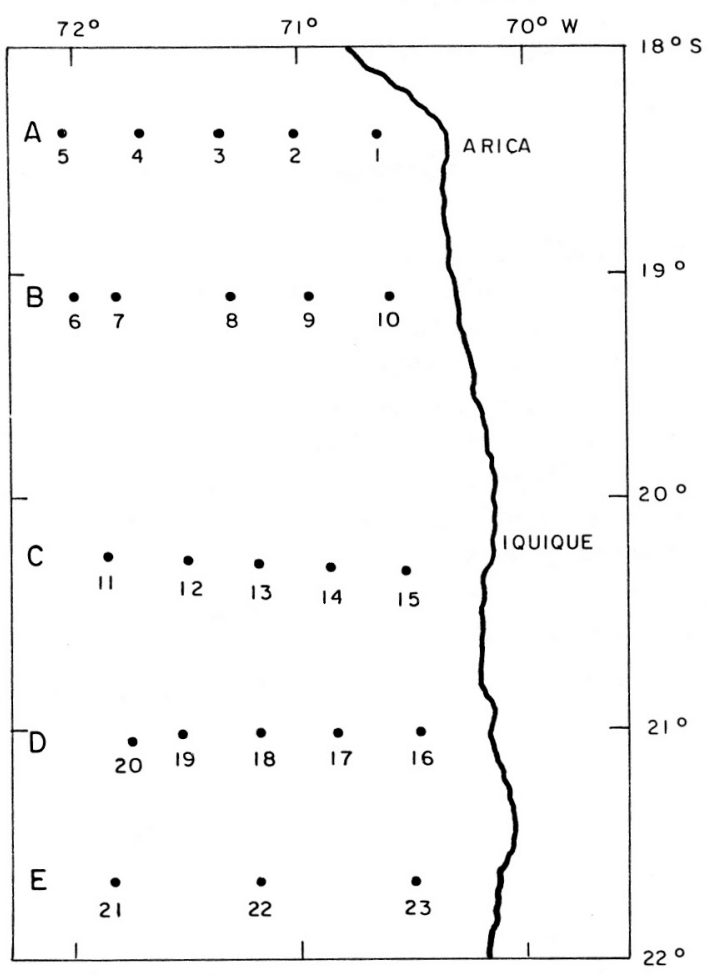

Figura 1. Ubicación de los transectos y estaciones de muestreo. 
Tabla 1. Fecha, ubicación geográfica, profundidad y duración de las faenas de pesca realizadas en las distintas estaciones de muestreo.

\begin{tabular}{|c|c|c|c|c|c|c|}
\hline $\begin{array}{l}\text { ESTACION } \\
\left(\mathrm{N}^{\circ}\right)\end{array}$ & $\begin{array}{l}\text { FECHA } \\
\text { (pesca) }\end{array}$ & $\begin{array}{l}\text { LATITUD } \\
\text { (S) }\end{array}$ & $\begin{array}{l}\text { LONGITUD } \\
\text { (W) }\end{array}$ & $\begin{array}{l}\text { PROFUNDI- } \\
\text { DAD (m) }\end{array}$ & $\begin{array}{c}\text { INICIO } \\
(\mathrm{hr})\end{array}$ & $\begin{array}{l}\text { TERMINO } \\
(\mathrm{hr})\end{array}$ \\
\hline 01 & $08 / 09 / 88$ & $18^{\circ} 25^{\prime}$ & $70^{\circ} 40^{\prime}$ & 530 & 03:30 & $05: 28$ \\
\hline 02 & $08 / 09 / 88$ & $18^{\circ} 25^{\prime}$ & $71^{\circ} 01^{\prime}$ & 643 & $07: 55$ & $09: 40$ \\
\hline 03 & $08 / 09 / 88$ & $18^{\circ} 25^{\prime}$ & $71^{\circ} 22^{\prime}$ & 603 & $11: 55$ & $\mathrm{nr}$ \\
\hline 04 & $08 / 09 / 88$ & $18^{\circ} 25^{\prime}$ & $71^{\circ} 43^{\prime}$ & 513 & $15: 55$ & $17: 18$ \\
\hline 05 & $08 / 09 / 88$ & $18^{\circ} 25^{\prime}$ & $72^{\circ} 04^{\prime}$ & 485 & $19: 55$ & $\mathrm{nr}$ \\
\hline 06 & $09 / 09 / 88$ & $19^{\circ} 09^{\prime}$ & $72^{\circ} 00^{\prime}$ & 450 & $02: 40$ & $\mathrm{nr}$ \\
\hline 07 & $09 / 09 / 88$ & $19^{\circ} 00$ & $71^{\circ} 39^{\prime}$ & 425 & 07:00 & $08: 50$ \\
\hline 08 & $09 / 09 / 88$ & $19^{\circ} 09^{\prime}$ & $71^{\circ} 18^{\prime}$ & 200 & $11: 20$ & 13:06 \\
\hline 09 & $09 / 09 / 88$ & $19^{\circ} 09^{\prime}$ & $70^{\circ} 58^{\prime}$ & 576 & $16: 55$ & $17: 13$ \\
\hline 10 & $09 / 09 / 88$ & $19^{\circ} 09^{\prime}$ & $70^{\circ} 36^{\prime}$ & 450 & $19: 40$ & $21: 06$ \\
\hline 11 & $10 / 09 / 88$ & $20^{\circ} 19^{\prime}$ & $71^{\circ} 52^{\prime}$ & 306 & $09: 50$ & $11: 15$ \\
\hline 12 & $10 / 09 / 88$ & $20^{\circ} 19^{\prime}$ & $71^{\circ} 31^{\prime}$ & 450 & $13: 30$ & 15:03 \\
\hline 13 & $10 / 09 / 88$ & $20^{\circ} 20^{\prime}$ & $71^{\circ} 11^{\prime}$ & 540 & $19: 00$ & $20: 20$ \\
\hline 14 & $10 / 09 / 88$ & $20^{\circ} 20^{\prime}$ & $70^{\circ} 58^{\prime}$ & 450 & $22: 55$ & $00: 20$ \\
\hline 15 & $11 / 09 / 88$ & $20^{\circ} 21^{\prime}$ & $70^{\circ} 31^{\prime}$ & 450 & 03:00 & $\mathrm{nr}$ \\
\hline 16 & $11 / 09 / 88$ & $21^{\circ} 04^{\prime}$ & $70^{\circ} 30^{\prime}$ & 450 & $10: 00$ & $11: 33$ \\
\hline 17 & $11 / 09 / 88$ & $21^{\circ} 04^{\prime}$ & $70^{\circ} 51^{\prime}$ & 450 & $15: 20$ & $16: 45$ \\
\hline 18 & $11 / 09 / 88$ & $21^{\circ} 04^{\prime}$ & $71^{\circ} 11^{\prime}$ & 450 & $19: 10$ & $20: 46$ \\
\hline 19 & $11 / 09 / 88$ & $21^{\circ} 04^{\prime}$ & $71^{\circ} 31^{\prime}$ & 374 & $22: 50$ & $00: 37$ \\
\hline 20 & $12 / 09 / 88$ & $21^{\circ} 05^{\prime}$ & $71^{\circ} 47^{\prime}$ & 576 & $02: 50$ & $04: 05$ \\
\hline 21 & $12 / 09 / 88$ & $21^{\circ} 47^{\prime}$ & $71^{\circ} 51^{\prime}$ & 261 & $09: 15$ & $10: 41$ \\
\hline 22 & $12 / 09 / 88$ & $21^{\circ} 41^{\prime}$ & $71^{\circ} 11^{\prime}$ & 378 & $03: 40$ & $04: 33$ \\
\hline 23 & $12 / 09 / 88$ & $21^{\circ} 41^{\prime}$ & $70^{\circ} 31^{\prime}$ & 378 & $21: 11$ & $22: 48$ \\
\hline
\end{tabular}

$\mathrm{nr}=$ no registrado

boca y un copo de mallas de 500 micras. En cada estación el arte se operó a una velocidad de 4 nudos y un tiempo de arrastre efectivo de 30 minutos, por lo que las capturas son comparables en términos de abundancia relativa y no requieren de estandarización.

En cubierta el material fue fijado en formol al $5 \%$. En el laboratorio se procedió a la identificación, recuento del número y peso de los individuos por estación y lance. Para la confección del listado se consideró la ordenación sistemática de Nelson (1984). Cabe señalar que las condiciones de deterioro por captura del ejemplar de Argentinidae no permiten una identificación precisa. Además, el material de las familias Serrivomeridae, Stomiidae y Melanostomiidae aún se encuentra bajo estudio. Todo el material recolectado fue depositado en la colección zoológica del Departamento de Ciencias 
del Mar de la Universidad Arturo Prat, de lquique (UAP(PC) y UAP(PO)).

\section{Análisis sobre la distribución}

El análisis faunístico y biogeográfico consideró la información sobre distribución por especie que señalan las siguientes fuentes: Baird (1986) Sternoptychidae; Bussing (1965) Trachychthyidae; Castle (1986) Nemichthyidae; Chiu y Markle (1990) Melanonidae; Ebeling (1962) Melamphaeidae; Ebeling y Weed (1963) Melamphaeidae; Garman (1899) Trachycthyidae; Gibbs (1986a y b) Stomiidae y Astronesthidae; Gon (1990a y b) Stomiidae y Gonostomatidae; Hulley (1986a, b y c) Anoplogastridae, Stylephoridae e ldiacanthidae; Kobylyanskiy (1985) Bathylagidae; Novikova (1972) Chauliodontidae; y Schaefer et al. (1986) Photichthyidae.

\section{Tratamiento estadístico de las muestras}

A partir del análisis de las muestras se confeccionaron tablas de abundancias relativas de las especies por estación, lo cual permitió la estimación de los siguientes índices ecológicos:

- Abundancia relativa: representa el porcentaje correspondiente de una especie o familia sobre el total, en número de individuos y biomasa.

- Diversidad de especies: se aplicó el índice de Shannon- Wiener, estimado como

$$
H^{\prime}=-\sum p_{i} \log _{2} p_{i}
$$

donde:

$\mathrm{p}_{\mathrm{i}}=\mathrm{n} / \mathrm{N}=$ probabilidad de importancia de un ejemplar de la i-ésima especie en el total de ejemplares

- Similitud: se contempló el empleó del análisis de «clusters», expresado en forma de dendrogramas, aplicando el método UPGMA que utiliza medias aritméticas (Menni y Goztonyi, 1982).

Los datos fueron ingresados al programa computacional ACOM (Navarro, 1984), empleando matrices de 23 estaciones por 39 especies, con información sobre abundancia (número de ejemplares).

Para la elección del índice se siguió a Sáiz (1980) y en la determinación del nivel de similitud a Arancibia (1988). Además se utilizó el índice de
Winer (similitud biocenótica), donde:

donde:

$$
S w=\sum x y / \sqrt{\sum x^{2} \sum y^{2}}
$$

$\mathrm{x}=$ valor de importancia de cada elemento en la condición A

$\mathrm{y}=$ valor de importancia de cada elemento en la condición $\mathrm{B}$

\section{Evaluación acústica}

Los mictófidos han sido reconocidos como componentes importantes de la capa de dispersión sónica profunda (DSL) (Barham, 1966; Backus et al., 1968; Linkowski, 1983; Pearcy y Laurs, 1966; Gjosaeter, 1981). Por esta razón, las capturas fueron acompañadas del registro continuo de la DSL, mediante el equipo SIMRAD instalado a bordo del B/I «Carlos Porter», con una frecuencia de $32 \mathrm{khz}$ y un alcance hasta $500 \mathrm{~m}$ de profundidad. Para los efectos del presente análisis sólo se consideraron los transectos B, C y D (Fig. 1), en los cuales también se mantuvo el registro entre las estaciones de pesca.

\section{RESULTADOS}

\section{Condiciones oceanográficas}

La distribución superficial de la temperatura, salinidad y densidad presenta durante el período de estudio un patrón característico, que consiste en un lente de agua desarrollado entre Arica y el Río Loa. Las particularidades de este lente son la isoterma de $18^{\circ} \mathrm{C}$ (Fig. 2), la isohalina de $34,5 \times 10^{-3}$ (Fig. 3) y la isopicna de 25 unidades de sigma-t (Fig. 4).

El diagrama T-S y el triángulo de mezcla de las estaciones oceanográficas ubicadas entre Arica y El Loa (Fig. 5), muestran al oeste de Arica la participación del agua subtropical. A su vez, se puede apreciar hacia el sector más costero una mezcla del tipo de agua subtropical y subantártica en los niveles superficiales. En el nivel de los $250 \mathrm{~m}$ de profundidad se encuentra un gran porcentaje de agua ecuatorial subsuperficial e intermedia antártica, acentuándose esta última en el nivel de los $500 \mathrm{~m}$ de profundidad. 


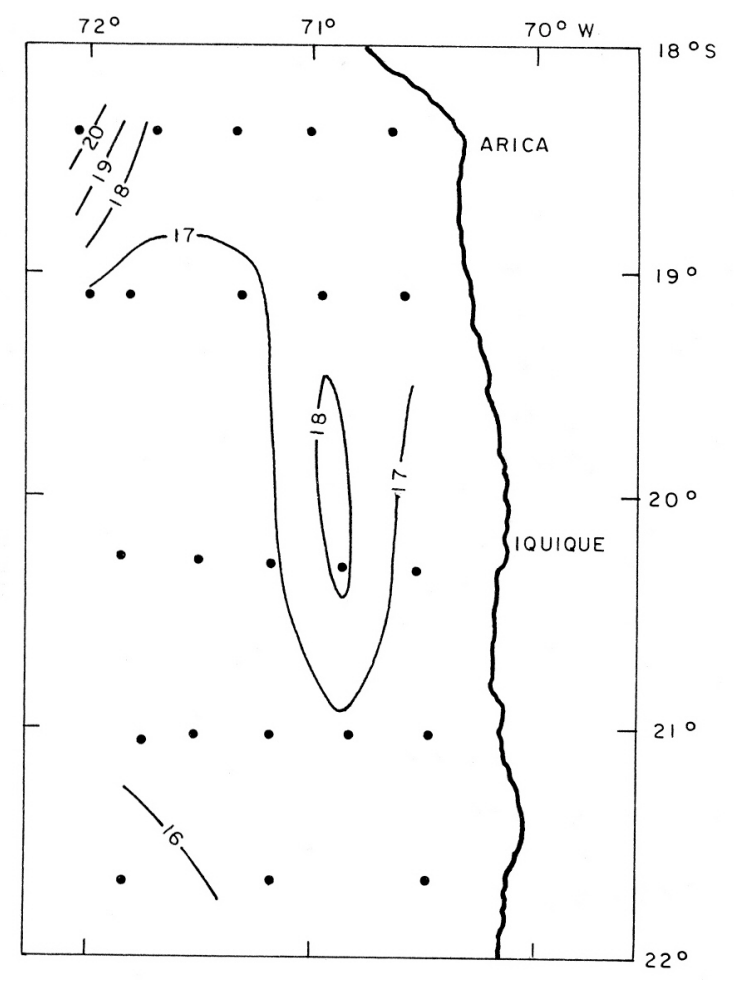

Figura 2. Temperatura superficial $\left({ }^{\circ} \mathrm{C}\right)$ del mar durante la prospección mesopelágica (septiembre 1988).

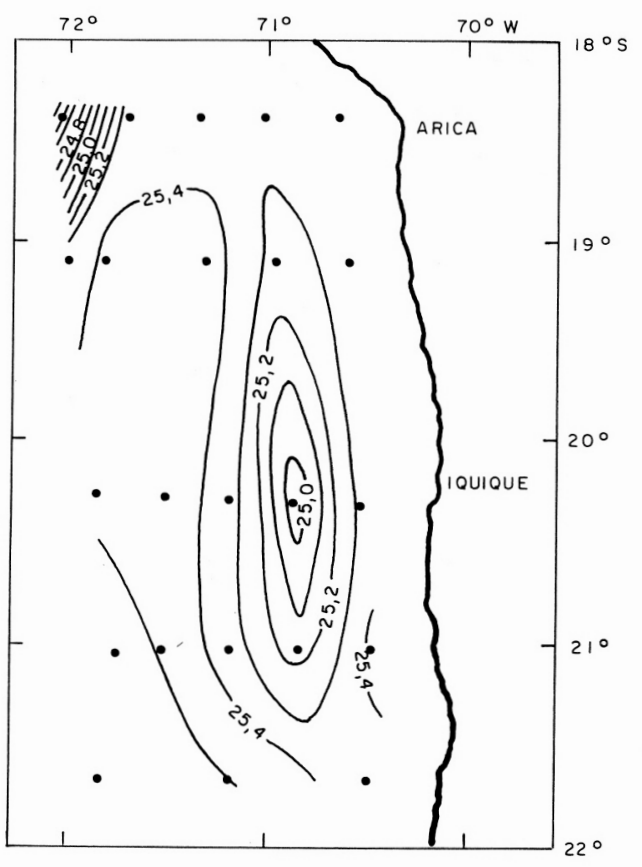

Figura 4. Densidad superficial del mar (sigma-t) durante la prospección mesopelágica (septiembre 1988).

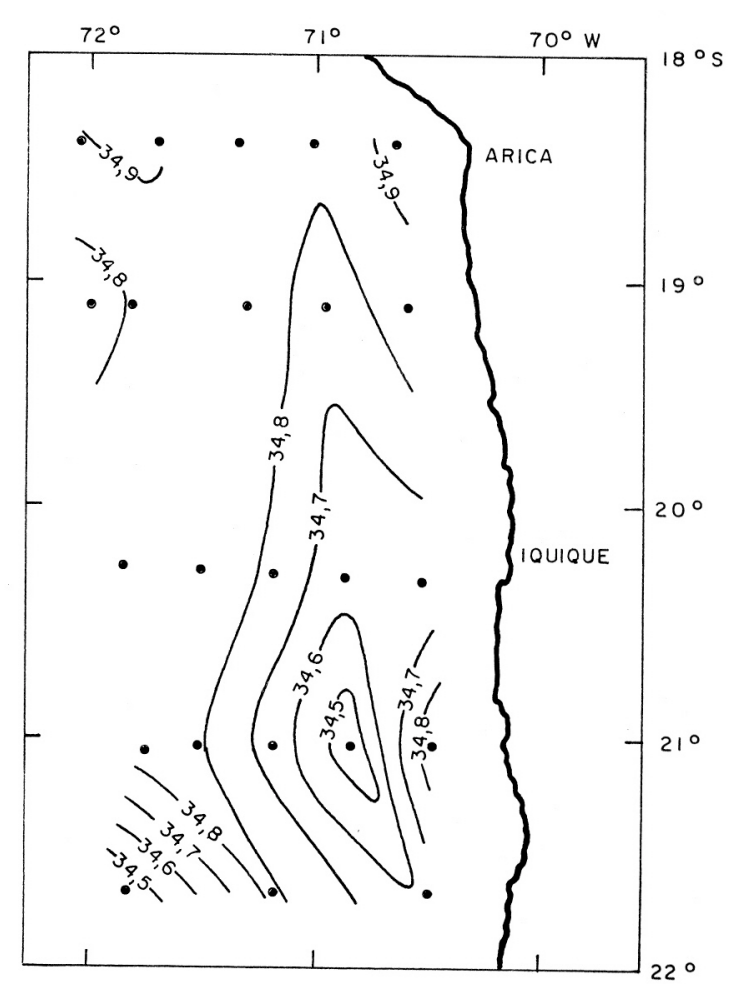

Figura 3. Salinidad superficial del mar $\left(\mathbf{S x 1 0 ^ { - 3 } )}\right.$ durante la prospección mesopelágica (septiembre 1988).

\section{Ictiofauna mesopelágica}

\section{Composición y abundancia}

En el área de estudio se obtuvo un total de 39 especies que pertenecen a 21 familias; se destacan Cyclothone acclinidens Garman, 1899 por su abundancia numérica $(62,13 \%)$, y Triphoturus mexicanus (Gilbert, 1890) por su biomasa (15,29\%), ambas con una frecuencia de ocurrencia por estación del 91,3\% (Tabla 2).

De las familias destacaron Gonostomatidae (62,13\% de la abundancia numérica) y Mycthophidae (24,69\% de la biomasa) (Fig. 6). Myctophidae y Melamphaidae fueron las familias mejor representadas, con 7 y 5 especies respectivamente (Tabla 2).

\section{Análisis por estación}

En el 32,8\% de las estaciones se registró entre 10 y 15 especies, destacando la estación 23 con 15 especies y la estación 12, con 2 especies, siendo la media estimada de 8,5 especies por estación (Fig. 7).

En el análisis de la abundancia numérica desta- 


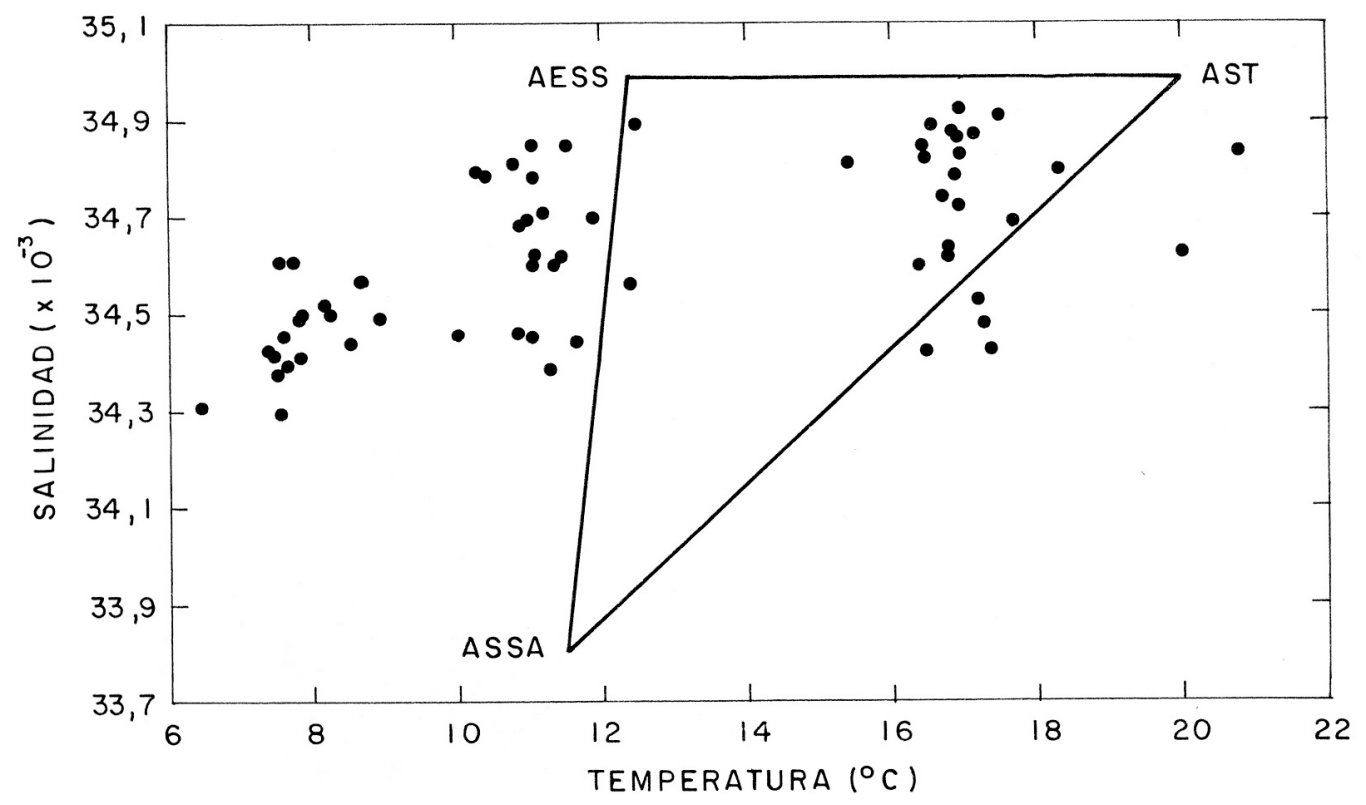

Figura 5. Diagrama T-S y triángulo de mezcla en la columna de agua $(0-1000 \mathrm{~m})$ en las estaciones oceanográficas (septiembre 1988).

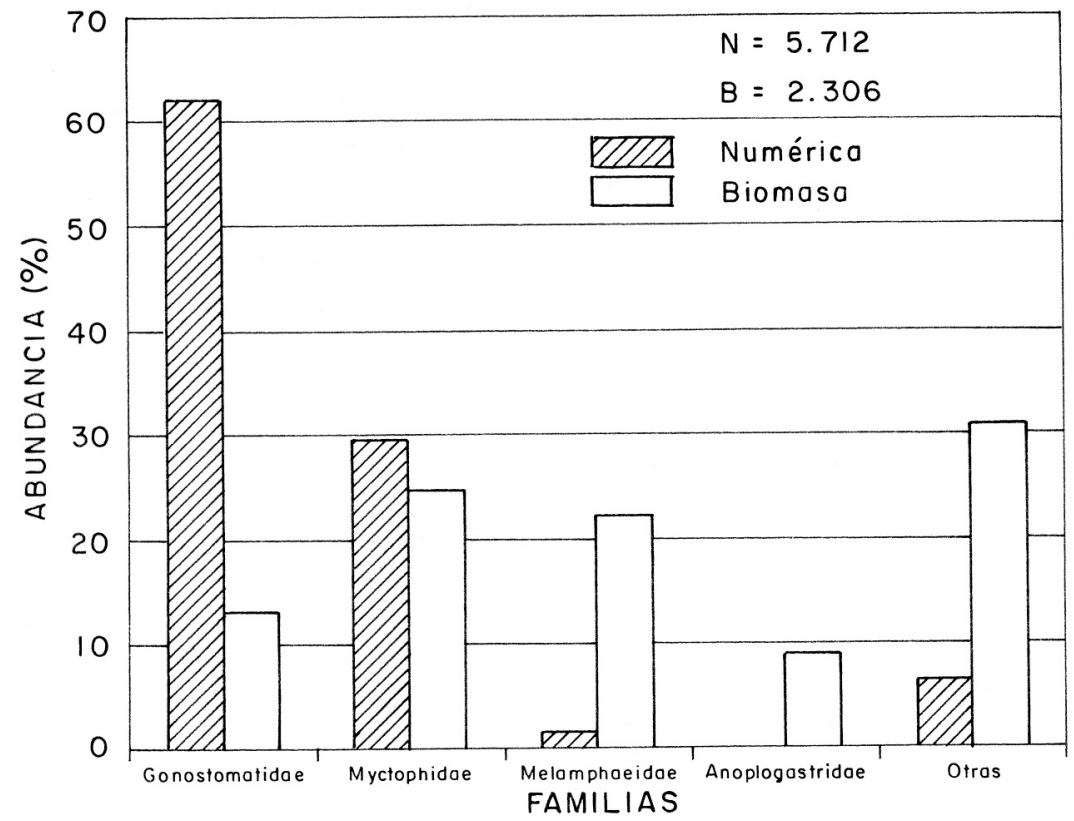

Figura 6. Abundancia en número y en peso de las familias de peces mesopelágicos más abundantes $(\mathrm{N}=$ número total de individuos capturados; $\mathrm{B}=$ biomasa total en $\mathbf{g}$ ). 
Tabla 2. Abundancia en número (n), biomasa (g) y frecuencia de ocurrencia de las especies de la ictiofauna mesopelágica.

\begin{tabular}{|c|c|c|c|}
\hline FAMILIAS & $\begin{array}{l}\text { INDIVIDUOS } \\
n \quad(\%)\end{array}$ & $\begin{array}{c}\text { BIOMASA } \\
\mathrm{g} \quad(\%)\end{array}$ & $\begin{array}{c}\text { FRECUENCIA } \\
\mathrm{n} \quad(\%)\end{array}$ \\
\hline SERRIVOMERIDAE Serrivomer $s p$. & $2(0,04)$ & $19,7(0,70)$ & $2(8,70)$ \\
\hline NEMICHTHYIDAE Nemichthys scolapaceus & $3(0,05)$ & $94,2(3,36)$ & $3(13,04)$ \\
\hline ARGENTINIDAE & $1(0,02)$ & $4,8(0,17)$ & $1(4,35)$ \\
\hline BATHYLAGIDAE $\quad$ Bathylagus nigrigenys & $15(0,26)$ & $25,8(0,92)$ & $11(47,83)$ \\
\hline Binghamnichthys microphos & $1(0,02)$ & $36,9(1,32)$ & $1(4,35)$ \\
\hline Talismania bifurcata & $5(0,09)$ & $30,9(1,10)$ & $1(4,35)$ \\
\hline Holtbyrnia macrops & $1(0,02)$ & $9,0(0,32)$ & $1(4,35)$ \\
\hline Cyclothone acclinidens & $3.549(62,13)$ & $368,2(13,12)$ & $21(91,30)$ \\
\hline PHOTICHTHYIDAE Vinciguerria lucetia & $254(4,45)$ & $58,4(2,08)$ & $19(82,61)$ \\
\hline STERNOPTYCHIDAE $\quad$ Argyropelecus affinis & $8(0,14)$ & $44,0(1,57)$ & $5(21,74)$ \\
\hline Argyropelecus lychnus & $8(0,14)$ & $15,2(0,54)$ & $5(21,74)$ \\
\hline Sternoptyx diaphana & $23(0,40)$ & $10,7(0,38)$ & $10(43,48)$ \\
\hline ASTRONESTHIDAE Astronesthes sp. & $1(0,02)$ & $3,6(0,13)$ & $1 \quad(4,35)$ \\
\hline Borostomias antarticus & $1(0,02)$ & $74,4(2,65)$ & $1(81,35)$ \\
\hline Indeterminatae & $7(0,12)$ & $24,6(0,88)$ & $17(73,91)$ \\
\hline MELANOSTOMIIDAE & $1(0,02)$ & $49,4(1,76)$ & $1(4,35)$ \\
\hline Indeterminatae 2 & $3(0,05)$ & $10,7(0,38)$ & $3(13,04)$ \\
\hline Indeterminatae 3 & $15(0,26)$ & $13,1 \quad(0,47)$ & $8(34,78)$ \\
\hline CHAULIODONTIDAE & $1(0,02)$ & $8,7(0,31)$ & $1(4,35)$ \\
\hline Chauliodus vasnetzovi & $9(0,16)$ & $66,6(2,37)$ & $6(26,09)$ \\
\hline IDIACANTHIDAE Idiacanthus $s p$. & $3(0,05)$ & $4,9(0,17)$ & $3(13,04)$ \\
\hline Diogenichthys atlanticus & $972(17,02)$ & $182,5(6,50)$ & $15(65,22)$ \\
\hline Diogenichthys laternatus & $4(0,07)$ & $7,5(0,27)$ & $4(17,39)$ \\
\hline Gonichthys sp. & $5(0,09)$ & $0,9(0,03)$ & $3(13,04)$ \\
\hline Hygophum hanseni & $1(0,02)$ & $1,2(0,04)$ & $1(4,35)$ \\
\hline Hygophum sp. & $1(0,02)$ & $0,8(0,03)$ & $1(4,35)$ \\
\hline Lampanyctus achirus & $4(0,07)$ & $71,1(2,53)$ & $3(13,04)$ \\
\hline Triphoturus mexicanus & $705(12,34)$ & $429,1(15,29)$ & $21(91,30)$ \\
\hline NEOSCOPELIDAE Scopelengys tristis & $3(0,05)$ & $11,0(0,39)$ & $2(8,70)$ \\
\hline STYLEPHORIDAE Stylephorus chordatus & $1(0,02)$ & $20,0(0,71)$ & $1(4,35)$ \\
\hline Melanonus zugmayeri & $1(0,02)$ & $97,0(3,46)$ & $1(4,35)$ \\
\hline MELAMPHAIDAE Melamphaes acanthomus & $11(0,19)$ & $153,6(5,47)$ & $2(8,70)$ \\
\hline Melamphaes spinifer & $3(0,05)$ & $4,9(0,17)$ & $3(13,04)$ \\
\hline Poromitra megalops & $1(0,02)$ & $1,5(0,05)$ & $1(4,35)$ \\
\hline Scopelogadus mizolepis & $40(0,70)$ & $339,9(12,12)$ & $13(56,52)$ \\
\hline Indeterminatae & $40(0,70)$ & $124,1(4,42)$ & $7(30,43)$ \\
\hline ANOPLOGASTRIDAE & $4(0,07)$ & $252,2(8,99)$ & $4(17,39)$ \\
\hline TRACHICHTYIDAE Trachichthys mento & $4(0,07)$ & $109,8(3,91)$ & $2(8,70)$ \\
\hline CARISTIIDAE $\quad$ Caristius japonicus & $1(0,02)$ & $24,7(0,88)$ & $1 \quad(4,35)$ \\
\hline $\begin{array}{llllllll} & O & T & A & L & E & S \\
\end{array}$ & $5.712(100)$ & $2.806(100)$ & $23 \quad(100)$ \\
\hline
\end{tabular}




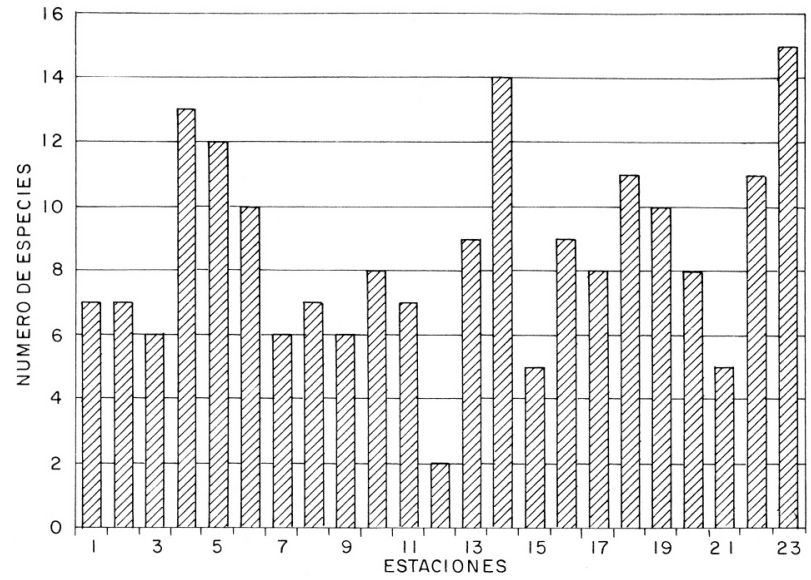

Figura 7. Número de especies capturadas por estación de muestreo.

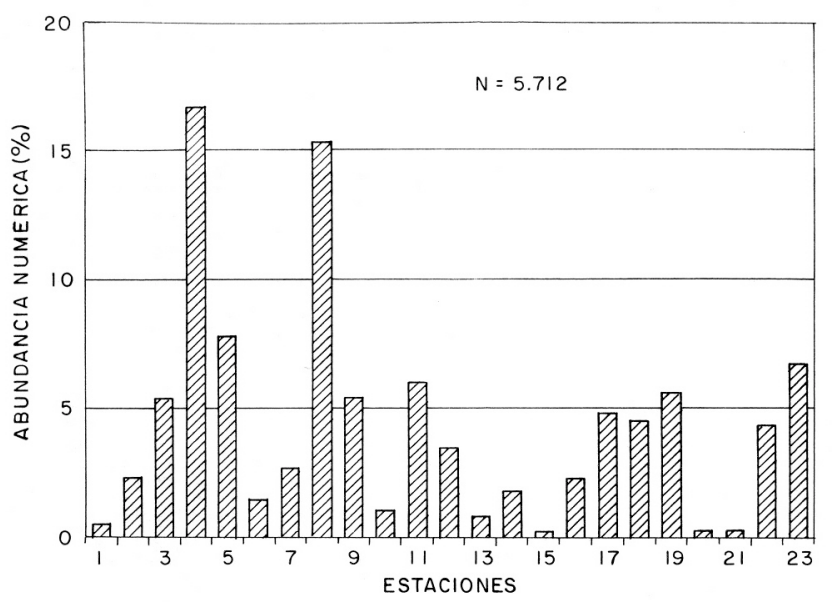

Figura 8. Porcentaje del número total de individuos por estación de muestreo ( $\mathrm{N}$ = número de individuos capturados).

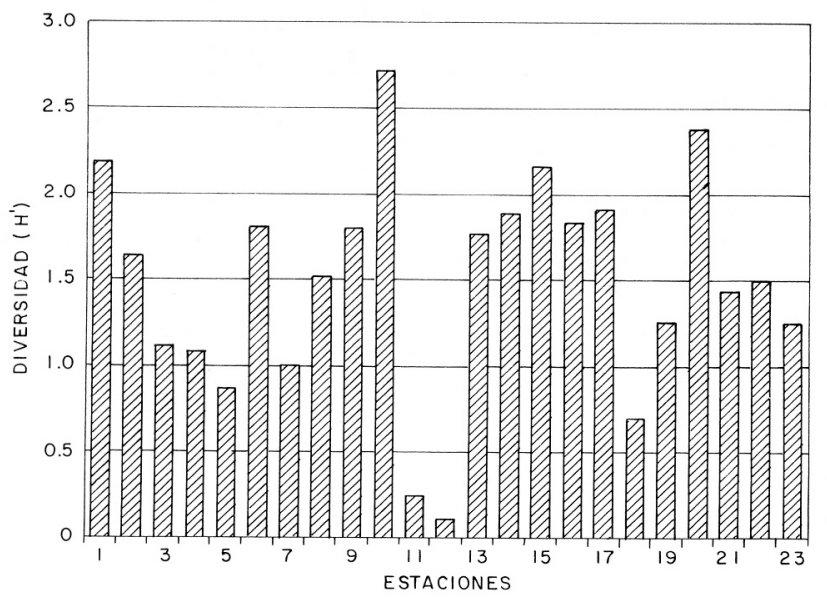

Figura 9. Diversidad específica (índice de Shannon-Wiener) por estación de muestreo. 
caron las estaciones 4 y 8 , ambas sobre el $13 \%$ del total; las abundancias correspondientes a las estaciones restantes fueron inferiores al 8\% (Fig. 8).

El índice de diversidad de Shannon- Wiener fluctuó entre 2,72 (estación 10) y 0,01 (estación 12), con una media de 1,49 (Fig. 9).

El análisis de similitud biocenótica muestra 4 grupos discretos a un nivel de similitud del $86 \%$; destaca el grupo que reúne 12 estaciones ubicadas en los $18^{\circ} 25^{\prime}-21^{\circ} 41^{\prime} \mathrm{S}$ y los $70^{\circ} 31^{\prime}-72^{\circ} 04^{\prime} \mathrm{W}$ (Figs. 1 y 10).

\section{Relaciones ictiofaunísticas}

Según las distribuciones por especie, el 61,5\% (25 spp.) de la ictiocenosis del área de estudio incluyó a especies típicas de aguas temperadas (tropicales y/o subtropicales) (Tabla 3). Este conjunto incluye especies preferentemente subtropicales (Chauliodus vasnetzovi Novikova 1972 y Melanonus zugmayeri Norman 1930), que parecen representar un aporte desde el oeste al área de estudio, así como especies típicamente tropicales (Melamphaes spinifer Ebeling 1962, Poromitra megalops (Lütken 1877),

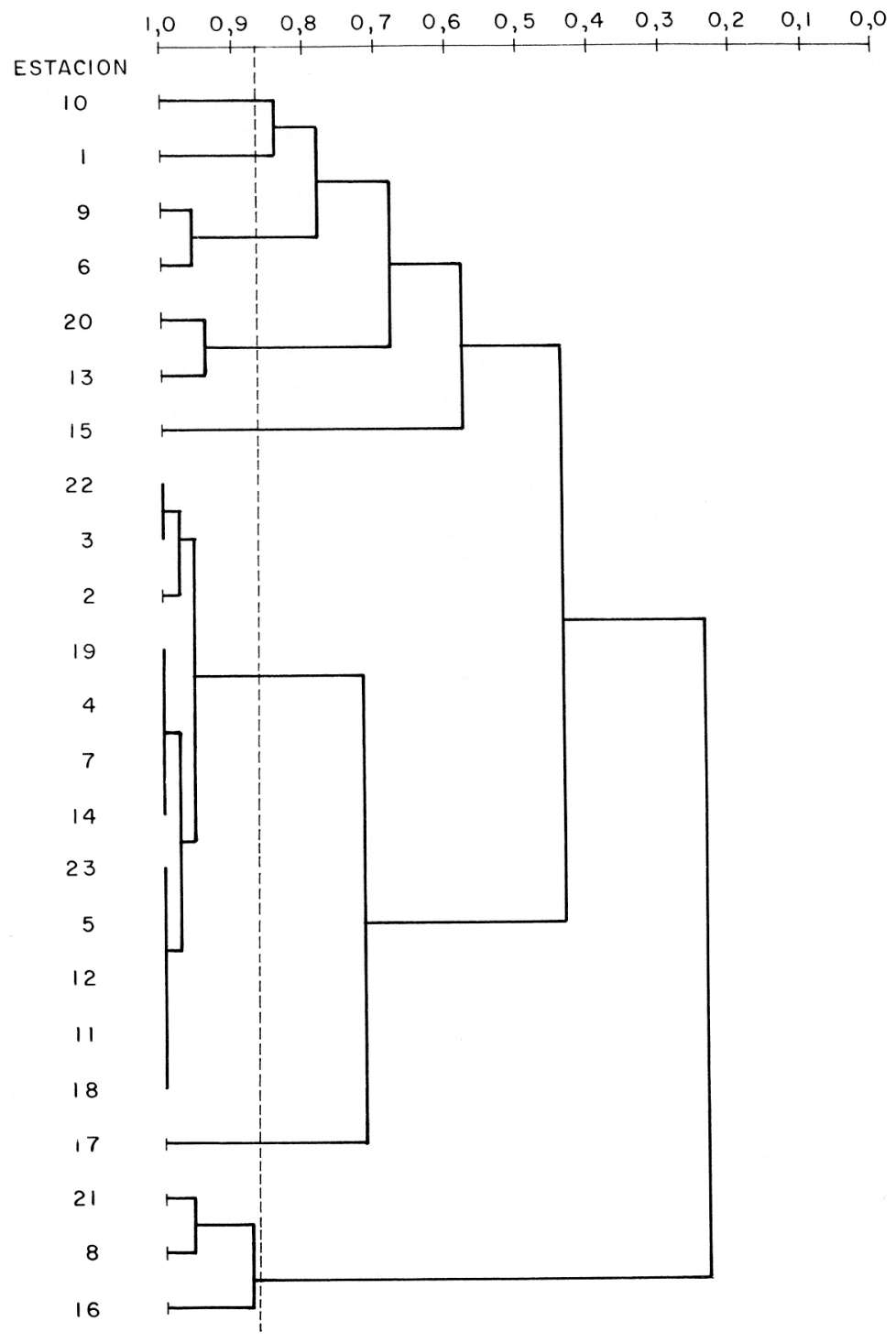

Figura 10. Similitud biocenótica (índice de Winer) entre estaciones de muestreo. 

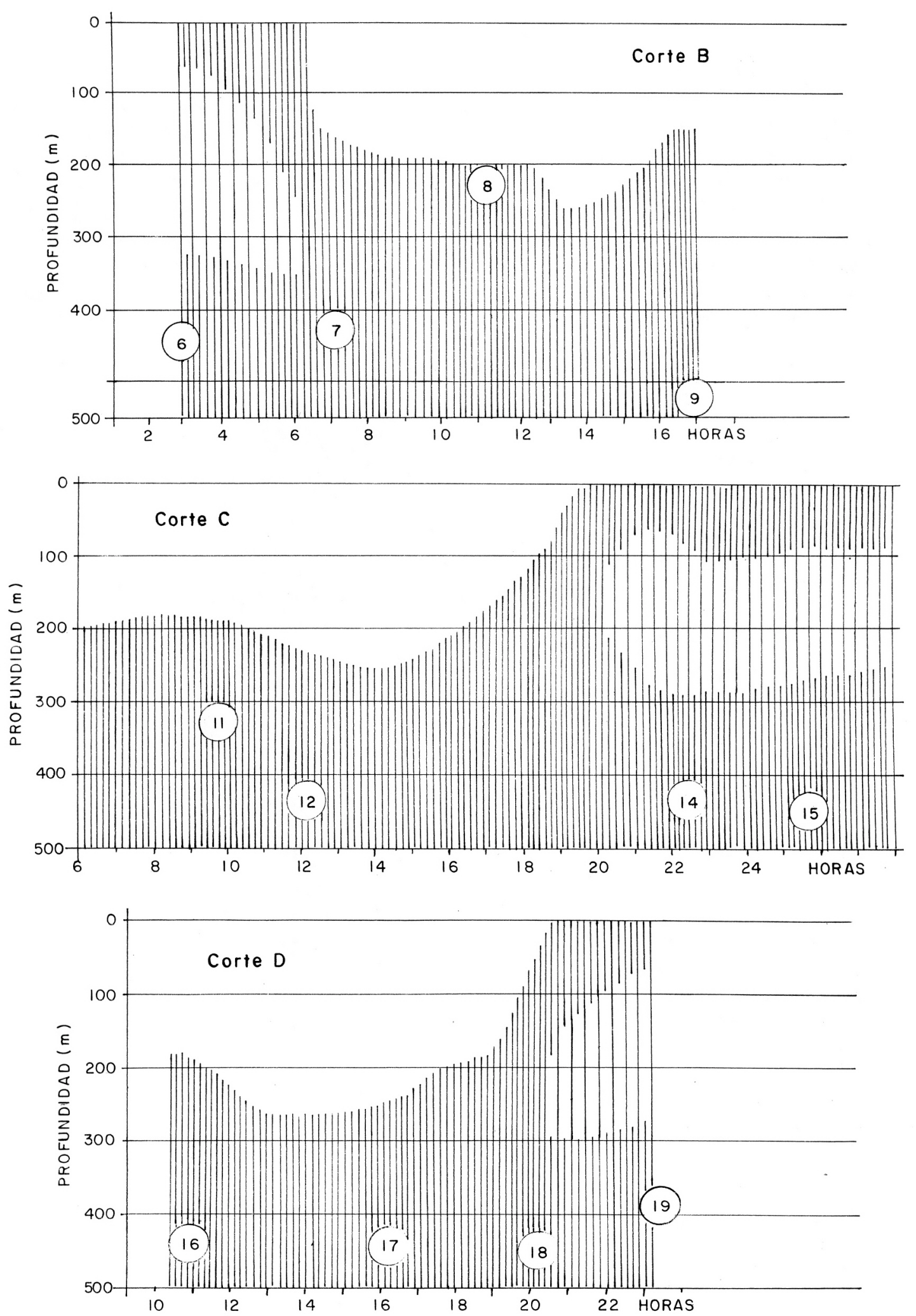

Figura 11. Comportamiento diario de la DSL en los cortes B, C y D durante la prospección de peces mesopelágicos. En círculo se indica el número de la faena de pesca realizada. 
Tabla 3. Distribución de las especies mesopelágicas capturadas en el presente estudio.

\begin{tabular}{|c|c|c|}
\hline ESPECIES & DISTRIBUCION & AUTOR \\
\hline Serrivomer & en todos los mares & Castle, 1986 \\
\hline Nemichthys scolapaceus Richardson, 1848 & tropical a subantártico & Castle, 1986 \\
\hline Bathylagus nigrigenys Parr, 1931 & tropical a subtropical & Kobylyanskiy, 1985 \\
\hline Binghamnichthys microphos (Parr, 1937) & $?$ & \\
\hline Talismanis bifurcata Parr, 1952 & tropical y subtropical & Kong y Meléndez, 1991 \\
\hline Holtbyrnia macrops Maul, 1957 & $?$ & \\
\hline Cyclothone acclinidens Garman, 1899 & tropical y subtropical & Gon, 1990 \\
\hline Vinciguerria lucetia (Garman, 1899) & tropical y subtropical & Schaefer et al., 1986 \\
\hline Argyropelecus lychnus Garman, 1899 & tropical y subtropical & Baird, 1986 \\
\hline Argyropelecus affinis Garman, 1899 & tropical y subtropical & Baird, 1986 \\
\hline Sternoptyx diaphana Hermann, 1781 & tropical y subtropical & Baird, 1986 \\
\hline Astronesthes & tropical y subtropical & Gibbs, $1986 b$ \\
\hline Borostomias antarticus (Lönnberg, 1905) & temperado y subantártico & Gon, 1990 \\
\hline Chauliodus sloani Bloch y Schneider, 1801 & tropical a subantártico & Gibbs, $1986 a$ \\
\hline Chauliodus vasnetzovi Novikova, 1972 & subtropical & Novikova, 1972 \\
\hline Idiacanthus & tropical y subtropical & Hulley, 1986a \\
\hline Diogenichthys atlanticus (Tänning, 1928) & tropical y subtropical & Sáez, 1982 \\
\hline Diogenichthys laternatus (Garman, 1899) & tropical y subtropical & Sáez, 1982 \\
\hline Gonichthys & $?$ & \\
\hline Hygophum hanseni (Tänning, 1932) & sur Convergencia Subantártica & Craddock y Mead, 1970 \\
\hline Lampanyctus achirus Andriashev, 1962 & circumaustral; Hemisferio Norte & Sáez, 1982 \\
\hline Triphothurus mexicanus Gilbert, 1890 & tropical y subtropical & Sáez, 1982 \\
\hline Scopelengys tristis Alcock, 1890 & $20-33^{\circ} \mathrm{N}$; tropical y subtropical & Hulley, 1984 \\
\hline Stylephorus chordatus Shaw, 1791 & tropical y subtropical & Hulley, 1986 \\
\hline Melanonus zugmayeri Norman, 1930 & sur Convergencia Subtropical & Chiu y Markle, 1990 \\
\hline Melamphaes acanthomus Ebeling, 1962 & tropical y subtropical & Ebeling, 1962 \\
\hline Melamphaes spinifer Ebeling, 1962 & tropical & Ebeling, 1962 \\
\hline Poromitra megalops (Lütken, 1877) & tropical & Ebeling y Weed, 1963 \\
\hline Scopelogadus mizolepis (Günther, 1878) & tropical & Ebeling y Weed, 1963 \\
\hline Anoplogaster cornuta (Cuv. y Val., 1833) & tropical y subtropical & Hulley, 1986 \\
\hline Trachichthys mento Garman, 1899 & tropical & Garman, 1899 \\
\hline Caristius japonicus Gill y Smith, 1905 & tropical a subantártico & Bussing, 1965 \\
\hline
\end{tabular}

$?=$ información puntual $\mathrm{y} / \mathrm{o}$ no concluyente. 
Scopelogadus mizolepis (Günther 1878) y Trachichthys mento Garman, 1899).

El 7,7\% (3 spp.) correspondió a peces de aguas frías (antárticas y/o subantárticas), que incluyen a Lampanyctus achirus Andriashev 1962, Hygophum hanseni (Tanning 1932) y probablemente Borostomias antarcticus (Lonnberg 1905); estas dos últimas fueron escasas (abundancia $<0,5 \%$; Tabla 2).

El 30,8\% restante (11 spp.) estuvo representado por: a) especies no identificadas de las familias Stomiidae (1 sp.), Melanostomiidae (3 spp.), Argentinidae (1 sp.), Myctophidae (Hygophum sp. y Gonichthys sp.) y Serrivomeridae (Serrivomer sp.); y b) especies de amplia distribución en el Pacífico (Caristius japonicus Gill y Smith, 1905; Binghamichthys microphos (Parr, 1937); y Holtbyrnia macrops Maul, 1957).

Estas especies, salvo aquellas de las familias Stomiidae y Melanostomiidae, sólo fueron acciden tales y estuvieron representadas por un ejemplar (Tabla 2). Las familias Stomiidae y Melanostomiidae (con 73,91\% y 52,17\% de frecuencia respectivamente; Tabla 2), son elementos batipelágicos integrados al estrato bajo de la comunidad mesopelágica. $\mathrm{Su}$ captura se realizó mayoritariamente en los lances de mayor profundidad en las estaciones 2 y 3 , y/o en aquéllos efectuados durante la noche y el alba en las estaciones 5, 6, 7, 14, 18, 19 y 23).

\section{Distribución batimétrica}

El registro ecográfico realizado a lo largo de los cortes B, C y D (Fig. 11), mostró un comportamiento diario característico y similar en los tres casos. Es así como durante el período de obscuridad (noche) los peces ascienden hasta la superficie, para posteriormente con el alba (alrededor de las $6 \mathrm{AM}$ ) iniciar un paulatino descenso hasta bajo los $200 \mathrm{~m}$ de profundidad, que culmina después del medio día (13:16; 14:00 y 14:00) (Fig. 11; cortes B, C y D respectivamente ).

Durante la tarde se inicia el ascenso que culmina alrededor de las 19 a $20 \mathrm{PM}$, proceso que aparentemente produce una baja en la densidad de individuos por expansión de la comunidad, lo cual permite explicar los menores rendimientos obtenidos en las capturas nocturnas (estaciones 1-3, 5, 11, 1520) (para horarios del lance de pesca ver Tabla 1).

La Fig. 11 muestra además que en el período de obscuridad se producen dos capas de dispersión sónica, separadas por una zona intermedia de menor dispersión. La capa superior presentó durante los períodos de obscuridad una distribución batimétrica desde 0 a 60-100 m; en tanto que la capa inferior se desarrolla bajo los $250 \mathrm{~m}$ y su límite inferior no fue, determinado en el presente estudio.

\section{DISCUSION}

Las condiciones oceanográficas registradas en el área presentaron el patrón típico de formación de un lente de agua con características similares a la evidencia encontrada para la zona por Robles et al. (1976). La ubicación del lente frente a Iquique es propio de una condición invernal. Del mismo modo, los diagramas T-S y el triángulo de mezcla de las estaciones entre Arica y El Loa, resultaron ser concordante con lo encontrado por Kelly et al. (1988).

La formación de grupos discretos (análisis de similitudes según el índice de Winer) muestra una relación directa entre las características de temperatura y salinidad del agua superficial del océano y la composición de la ictiofauna mesopelágica, particularmente en la formación del grupo de estaciones $2,3,4,5,7,11,12,14,18,19,22$ y 23 , cuya ubicación coincide con el lente de aguas tibias presente en el área. Este grupo de estaciones se caracterizó por la alta abundancia numérica de Cyclothone acclinidens $($ sobre $66 \%$; media = $79,6 \%$ ), aspecto que incide en una consecuente baja diversidad.

La media de las diversidades por estación (H' $=1,5$ ), representa un intermedio entre valores de H' = 1,3 de comunidades ícticas de la Antártica y 2-3 de comunidades ícticas tropicales señaladas por Targett (1981). Si bien el presente valor es menor que el observado por Arancibia (1992) para Chile Central $\left(\mathrm{H}^{\prime}=1,6\right)$, debe considerarse que este último corresponde a un conjunto de peces demersales, donde la heterogeneidad del sustrato juega un rol fundamental en la diversidad (Ojeda, 1981).

La distribución batimétrica de los peces mesopelágicos concuerda con lo señalado anteriormente por Tont (1975), permitiendo establecer la existencia de una capa superior migratoria y otra inferior estática que se confundiría en los niveles profundos con la comunidad batipelágica. Estos resultados se oponen, sin embargo, notablemente con el esquema de distribución encontrado por Gjosaeter (1981) en el mar de Arabia, donde dos 
capas sónicas diurnas se confundirían durante el período nocturno, por ascenso de la inferior.

En todos los registros del presente estudio se encontró en los primeros $50 \mathrm{~m}$ de la columna de agua una capa de dispersión sónica débil, que fue interpretada a partir de las capturas como zooplancton (fundamentalmente eufáusidos). Durante el período nocturno esta capa se confundió con la capa ascendente de peces mesopelágicos.

El análisis de la distribución batimétrica por estación indica que las migraciones afectan a las familias definidas como numéricamente más importantes: Myctophidae y Gonostomatidae.

Las capturas sólo nocturna, y/o los lances más profundos, de Neoscopelidae, Alepocephalidae, Stomiidae, Melanostomiidae, Serrivomeridae, Searsiidae y Anoplogasteridae, indican que estas familias corresponden a un estrato más profundo que los 300-500 $\mathrm{m}$ prospectados.

La comunidad íctica estudiada presentó mayoritariamente especies de aguas cálidas, siendo escasas las de aguas frías (ver abundancia numérica y biomasa en Tabla 2). No obstante su marcado caracter tropical, ésta no pierde su calidad de comunidad transicional entre las, de características subantárticas y tropicales. Esta estructura marca también claras diferencias con la comunidad mesopelágica, descrita por Craddock y Mead (1970) frente a los 30³4'S, quienes citan 65\% de especies de tipo subtropical y sólo Triphoturus mexicanus como especie tropical. En esta comparación debe considerarse la diferencia estacional de las recolecciones: las de estos autores son de verano (4 enero-4 febrero '66) y las del presente estudio de una situación invernal (3-12 septiembre' 88 ).

La comparación de la estructura específica de ambas comunidades mesopelágicas muestra escasa afinidad a nivel de especies, siendo comunes Nemichthys scolopaceus Richardson, 1848, Cyclothone acclinidens Garman, 1899, Vinciguerria luce tia (Garman, 1899), Argyropelecus affinis Garman, 1899, Sternoptyx diaphana Hermann, 1781, Diogenichthys atlanticus (Tiinning, 1928), Hygophum hanseni (Tiinning, 1932), Triphothurus mexicanus Gilbert, 1890, Melamphaes spinifer Ebeling, 1962, Scopelogadus mizolepis (Günther, 1878) y Caristius japonicus Gill y Smith, 1905. El alto número de especies (133) citado por Craddock y
Mead (1970), tiene relación con una mayor profundidad de muestreo, el que alcanzó hasta los 1000 $\mathrm{m}$.

\section{REFERENCIAS BIBLIOGRAFICAS}

Arana, P. y V. Gaete. 1983. La Pesca en el Océano Pacífico (1964-1980). In: Recursos Marinos del Pacífico. P. Arana (Ed.), U.S. Inter. Sea Grant Program y Escuela de Ciencias del Mar, UCV, Valparaíso: 216-252.

Arancibia, H. 1988. Cluster analysis: the use of a simple statistical method in the identification of groups. ICES, C.M.ID: 12 Statistics Cttee., Ref. Demersal Fish Cttee., 18 pp.

Arancibia, H. 1992. Distribution patterns of the demersal fish assemblage off Central Chile. Biología Pesquera, 21: 43-53.

Acuña, E. 1986. El recurso mictófidos (Pisces, Myctophidae): antecedentes en aguas chilenas y marco de referencia para su investigación. In: La Pesca en Chile. P. Arana (Ed.), Escuela de Ciencias del Mar, UCV, Valparaíso: 315-338.

Backus, R.H., J.E. Craddock, R.C. Haedrich, D.L. Shores, J.M. Teal, G.W. Mead y W.D. Claecke. 1968. Ceratoscopelus maderensis: peculiar sound scattering layer identified with this myctophid fish. Science, 160: 991-993.

Baird, R.C. 1986. Tribe Sternoptychini. In: Smith's Sea Fishes. M.M. Smith y P.C. Heemstra (Eds.), Springer Verlag, page 255.

Barham, E.G. 1966. Deep scattering layer migration and composition: observation fram a diving saucer. Science, 151: 1399-1403.

Bussing, W. 1965. Studies of the midwater-fishes of the Pero-Chile trench. Am. Geophys. Un., 1297: 185-227.

Castle, P.H.J. 1986. Family Nemichthyidae. In: Smith's Sea Fishes. M.M. Smith y P.e. Heemstra (Eds.), Springer Verlag, page 193.

Chiu, T.S. y D.F. MarkIe. 1990. Melanonidae In: Fishes of the Southern Ocean. O. Gon y P.C. Heemstra (Eds.), J.L.B. Smith Institute of Ichthyology, Grahamstown, South Africa: 188190.

Craddock, J.E. y G. W. Mead. 1970. Midwater fishes 
fishes from the Eastern South Pacific Ocean. Anton Bruun Report, 3: 1-46.

Ebeling, A.W. 1962. Melamphaidae. I Systematics and zoogeography of the species in the bathypelagic fish genus Melamphaes Günther. Dana Report, 58: $1-164$.

Ebeling, A.W. y H. Weed. 1963. Melamphaidae. III Systematics and distribution of the species in the bathypelagic fish genus Scopelogadus Vaillant. Dana Report; 60: 1-58.

Garman, S. 1899. Reports on an exploration off the west coasts of Mexico, Central and South America, and off the Galapagos Islans. Mem. Mus. Com. Zool. Harv. Coll., 24: 1-431.

Gibbs, R.H. Jr. 1986a. Family: Stomiidae. In: Smith's Sea Fishes. M.M. Smith y P.C. Heemstra (Eds.), Springer Verlag, page 229.

Gibbs, R.H. Jr. 1986b. Family: Astronesthidae. In: Smith's Sea Fishes. M.M. Smith y P.C. Heemstra (Eds.), Springer Verlag: 231-233.

Gjosaeter, J. 1981. Abundance and production of lantern-fish (Myctophidae) in the western and northem Arabian Sea. Fisk. Dir. Ser. Hav. Unders., 17: $215-251$

Gjosaeter, J. y K. Kawaguchi. 1980. A review of the world resources of mesopelagic fish. FAO Fish. Tecn. Pap., 193: 1-151.

Gon, O. 1990a. Stomiidae. In: Fishes of the Southem Ocean. O. Gon y P.C. Heemstra (Eds.), J.L.B. Smith Institute of Ichthiology, Grahamstown, South Africa: $127-133$.

Gon, O. 1990b. Gonostomatidae. In: Fishes of the Southern Ocean. O. Gon y P.C. Heemstra (Eds.), J.L.B. Smith Institute of Ichthiology, Grahamstown, South Africa: 116-122.

Hulley, P.A. 1984. Neoscopelidae. In: Fishes of the North-Eastern Atlantic and the Mediterranean. P. Whitehead, M. Bauchot, J. Hureau, J. Nielsen y E. Tortonese (Eds.), UNESCO, Paris, 1: 426-428.

HuUey, P.A. 1986a. Family Idiacanthidae. In: Smith's Sea Fishes. M.M. Srnith y P.C. Heemstra (Eds.), Springer Verlag, page 234.

Hulley, P.A. 1986b. Family Anoplogasteridae. In:
Smith's Sea Fishes. M.M. Smith y P.C. Heemstra (Eds.), SpringerVerlag, page 415.

Hulley, P.A. 1986c. Family Stylephoridae. In: Smith's Sea Fishes. M.M. Smith y P.C. Heemstra (Eds.), Springer Verlag, page 404.

Kelly, R., R. Rubio y J. Osses. 1988. Condiciones oceanográficas en la zona norte de Chile, en invierno de 1988. In: Cuantificación de la biomasa de recursos pelágicos y vigilancia biooceanográficas zona norte (agosto 1988). Programa de Investigaciones Pesqueras de Recursos Pelágicos-Zona Norte. IFOP, Santiago, Chile, 16-46 pp.

Knox, G. 1970. Biological oceanography of the South Pacifico In: Scientific Exploration of the South Pacifico W.S. Wooster (Ed.), National Academy of Sciences, Washington DC: 155-182.

Kobylyanskiy, S.G. 1985. Material for the revision of the genus Bathylagus Günther (Bathylagidae): the group of «light» Deepsea Smelts. J. Ichthyol., 25(2): 1-17.

Kong, I. y R. Meléndez. 1991. Estudio taxonómico y sistemático de la ictiofauna de aguas profundas capturada entre Arica e Isla Mocha (18 $30^{\prime}-$ $38^{\circ} 30^{\prime}$ S). Estud. Oceanol., 10: 1-81.

Linkowsky, T.B. 1983. Electrona carlsbergi (Täning 1932) the principal component of a deep scattering layer in the Pacific sector of the Antarctic Ocean. Polar Res., 4: 71-78.

Menni, R. y A. Gostonyi. 1982. Benthic and semidemersal fish associations in the Argentine Sea. Studies Neotropical Fauna and Environment, 17: 1-29.

Millero F.J. y A. Poisson. 1981. International oneatmosphere equation of state of seawater. DeepSea Res., 28: 625-629.

Navarro, R. 1984. Programa computacional para análisis numérico de comunidades: diversidad y sobreposición. Medio Ambiente, 7: 82-87.

Nelson, J. 1984. Fishes of the world. John Wiley \& Sons., Inc., 523 pp.

Novikova, N. 1972. A new species of the genus Chau/iodus (Pisces, Chauliodontidae) from the Southeastern Pacifico J. Ichthyol., 12(1): 34-40. 
Ojeda, P. 1981. Estructura comunitaria de peces demersales en el extremo austral de Chile: explicación ecológica de patrones latitudinales, batimétricos y de simpatría. Tesis, Facultad de Ciencias Básicas y Farmacológicas, Universidad de Chile, $91 \mathrm{pp}$.

Parín, N.V., G.A. Golovan, N.P. Pakhorukov, Y.I. Sazonov y Y.I. Sherbachev. 1980. Fishes from the Nazca and Sala y Gomez underwater ridges colJected in cruise of R/V «Ikhtiandr». In: Fishes ofthe Opean. N.V. Parín (Ed.), Sea. Acad. Se., P.P. Shirshov Inst. of Oceanol., Moscow, 5-18.

Pearcy, W.G. y R.M. Laurs. 1966. Vertical migration and distribution of mesopelagic fishes off Oregon. Deep-Sea Res., 13: 153-165.

Robles, F., E. Alarcón y A. Ulloa. 1976. Las masas de agua en la región norte de Chile y sus variaciones en un período frío (1967) y en períodos cálidos (1969, 1971-73). FAO Inf. Pesca, 185: 94196.

Sáez, L.E. 1982. Myctophidae (Pisces, Osteichthyes, Myctophiformes) recolectados por la expedición «Itzumi-Pelágico I», enero-febrero 1980. Tesis Univ. de Concepción, 100 pp.

Recibido el 20 de julio de 1995.

Aceptado el 18 de diciembre de 1995.
Sáiz, F. 1980. Experiencias en el uso de criterios de similitud en el estudio de comunidades. Arch. Biol. Méd. Exp., 13: 387-402.

Schaefer, S., R.K. Johnson y J. Badcok. 1986. Family Photichthyidae. In: Smith's Sea Fishes. M.M. Smith y P.C. Heemstra, (Ed.s), Springer Verlag, 244-253.

Sverdrup, H.U., M.W. Johnson y R.H. Fleming. 1942. The Oceans. Prentice-HalJ, NewYork, 1087 pp.

Target, T.E. 1981. Trophic ecology and structure of coastal Antarctic fish communities. Marine Ecology Progress Series 4: 243-263.

Tont, S.A. 1975. Deep scattering layers: pattems in the Pacifico CALCOFI Rep., 18: 112-117.

Wisner, R.L. 1976. The taxonomy and distribution of lantemfishes (Family Myctophidae) of the eastem Pacific Ocean. Navy Ocean Research and Development Activity, Mississippi, NORDA Report, 3: 1-229 pp. 